\title{
The efficacy of daily snack replacement with oligofructose-enriched granola bars in overweight and obese adults: a 12-week randomised controlled trial
}

\author{
Korrie Pol $^{1}$, Cees de Graaf ${ }^{1}$, Diederick Meyer ${ }^{2}$ and Monica Mars ${ }^{1 *}$ \\ ${ }^{1}$ Division of Human Nutrition, Wageningen University \& Research, 6700 AA Wageningen, The Netherlands \\ ${ }^{2}$ Sensus, Borchwerf 3, 4704 RG Roosendaal, The Netherlands \\ (Submitted 11 May 2017 - Final revision received 20 December 2017 - Accepted 11 January 2018 - First published online 1 March 2018)
}

\section{Abstract}

Oligofructose is a prebiotic dietary fibre obtained from chicory root inulin. Oligofructose supplementation may affect satiety, food intake, body weight and/or body composition. The aim was to examine the efficacy of oligofructose-supplemented granola bars on the following weight management outcomes: satiety, energy intake, body weight and body composition in overweight or obese adults. In all, fifty-five adults with overweight or obesity (thirty-six females/nineteen males; age: 41 (SD 12) years; 90.6 (SD 11.8) kg; BMI: 29.4 (sD 2.6) kg/m²) participated in a parallel, triple-blind, placebo-controlled intervention. A total of twenty-nine subjects replaced their snacks twice a day with an equienergetic granola bar supplemented with $8 \mathrm{~g}$ of oligofructose (OF-Bar). Subjects in the control group $(n$ 26) replaced their snack with a control granola bar without added oligofructose (Co-Bar). Satiety, 24-h energy intake, body weight and body composition (fat mass and waist circumference) were measured at baseline, weeks 6 and 12. In addition, weekly appetite and gastrointestinal side effects were measured. During the intervention, energy intake, body weight and fat mass remained similar in the Co-Bar and OF-Bar groups (all $P>0.05$ ). Both groups lost 0.3 (sD 1.2) kg lean mass $(P<0 \cdot 01)$ and reduced their waist circumference with $-2 \cdot 2(\mathrm{sD} 3 \cdot 6) \mathrm{cm}(P<0 \cdot 0001)$ after 12 weeks. The OF-Bar group reported decreased hunger in later weeks of the intervention $(P=0.04)$, less prospective food consumption $(P=0.03)$ and less thirst $(P=0.003)$. To conclude, replacing daily snacks for 12 weeks with oligofructose-supplemented granola bars does not differentially affect energy intake, body weight and body composition compared with a control bar. However, there was an indication that appetite was lower after oligofructose bar consumption.

Key words: Dietary supplements: Obesity: Overweight: Oligofructose: Inulin-type fructan: Body composition: Food intake: Satiety

Observational data show that fibre-rich diets are associated with lower body weight or $\mathrm{BMI}^{(1,2)}$. Moreover, experimental studies have shown that foods naturally rich in dietary fibres may result in earlier satiation ${ }^{(3)}$ and suppressed hunger feelings ${ }^{(4,5)}$. A strategy for body weight management might therefore be to increase dietary fibre intake through fibre-supplemented foods that promote satiety or dilute energy density. However, dietary fibres are a heterogeneous group of compounds, some of which lower body weight and some of which do not influence appetite or body weight ${ }^{(6,7)}$.

A dietary fibre that has been often studied in relation to body weight management is oligofructose. Oligofructose is a soluble inulin-type fructan with a degree of polymerisation of 2-8. It has a sweet taste and may be used to replace sugar in sweet foods ${ }^{(8,9)}$. Oligofructose is neither digested nor absorbed in the small intestine and therefore delivers less energy - that is, $6.3 \mathrm{~kJ} / \mathrm{g}(1.5 \mathrm{kcal} / \mathrm{g})$ instead of $17 \mathrm{~kJ} / \mathrm{g}(4 \mathrm{kcal} / \mathrm{g})$ - compared with fully digestible carbohydrates ${ }^{(10)}$. This fibre is obtained from chicory root inulin ${ }^{(11)}$ and is well known for its prebiotic effect $^{(11-13)}$

Several animal studies have shown that oligofructose supplementation lowers energy intake (EI), prevents body weight gain and improves body composition ${ }^{(14-20)}$. However, the evidence from human data on food intake tends to be contradictory. Several short-term clinical studies have found effects of oligofructose on satiety feelings and food intake ${ }^{(21)}$, whereas others found only effects on satiety ${ }^{(22,23)}$ or food intake ${ }^{(24,25)}$, or no effects at all ${ }^{(26,27)}$.

It has been hypothesised that oligofructose triggers weight loss or maintains body weight because it decreases food intake. This decrease may be due to a modified satiety (hormone) response ${ }^{(28)}$. Oligofructose may influence satiety and food intake through fermentation by the gut microbiota; oligofructose can selectively stimulate growth and activity of the gut bacteria Bifidobacterium genus, which are associated with health ${ }^{(12)}$. However, a longer study period is required in order

Abbreviations: BSA, body surface area; Co-Bar, control granola bar; EI, energy intake; FM, fat mass; OF-Bar, oligofructose granola bar.

* Corresponding author: M. Mars, email monica.mars@wur.nl 
to observe beneficial health effects such as reduced body weight due to lower EI.

To date, several long-term parallel trials have investigated oligofructose supplementation on weight management outcomes $^{(28-30)}$. One study conducted a 12-week trial with forty-eight overweight and obese adults providing $21 \mathrm{~g} / \mathrm{d}$ oligofructose compared with maltodextrin. This study showed a $29 \%$ reduction in EI after 6 weeks and $1 \mathrm{~kg}$ reduction in body weight after 12 weeks. However, subjects did not report differences in appetite and satiety ${ }^{(28)}$. A shorter study performed an 8-week intervention with $30 \mathrm{~g} / \mathrm{d}$ oligofructose in twenty-two similar subjects. The results showed no effect on body weight and EI, but they did demonstrate that their subjective hunger and motivation to eat were reduced in the oligofructose group ${ }^{(29)}$. Recently, a trial with pre-diabetic subjects ( $n$ 44) showed that 18 -week consumption of $30 \mathrm{~g} / \mathrm{d}$ oligofructose-enriched inulin reduced body weight and food intake, but showed no effect on appetite $^{(30)}$. Thus, the aforementioned studies give inconsistent outcomes: in some cases positive effects on body weight and EI, whereas other studies affect appetite alone. The most important differences between the studies are the duration of supplementation, the study population and the dosage. That said, none of the long-term studies provided oligofructose in a real product, but instead used prepacked sachets that were added to foods or drinks.

In the present study, we investigated the efficacy of $16 \mathrm{~g}$ of oligofructose supplementation on several weight management outcomes, including satiety, EI, body weight and body composition. We hypothesised that subjects replacing their daily snacks with bars supplemented with oligofructose would have increased satiety feelings and have lower EI, which would result in a lower body weight and lower body fat mass (FM) compared with subjects replacing snacks with regular bars.

\section{Methods}

\section{Subjects}

In total, sixty-two volunteers with overweight or obesity (age: 20-60 years; BMI $\geq 25$ and $\leq 35 \mathrm{~kg} / \mathrm{m}^{2}$ ) were recruited from Wageningen and surroundings. The flow diagram of the inclusion of subjects can be found in Fig. 1. Inclusion criteria were apparently healthy as measured by questionnaire (i.e. not reported CVD or abnormalities, liver or pancreas disease, renal disease, thyroid disease, diabetes, stomach or bowel diseases, unstable hypertension or unstable dyslipidaemia), and no use of medicines judged likely to interfere with the experiment in the preceding 3 months to study entry (e.g. appetite or EI suppressing drugs, antibiotics, bulk laxatives). Participants who reported weight fluctuations $>5 \mathrm{~kg}$ for the last 3 months and those following a diet or exercise regimen, or being excessively physically active, were excluded from the study. Participants were required to have a normal appetite and to regularly consume snacks. Participants were excluded if they were allergic to the study food, followed a special diet (e.g. vegetarian), consumed $\geq 3$ alcoholic drinks/d on average, were pregnant or breast-feeding. In addition, individuals were excluded if they had a fasting glucose concentration $>6.9 \mathrm{mmol} / 1$ or fasting total cholesterol concentration $>6.5 \mathrm{mmol} / \mathrm{l}$ or fasting TAG concentration $>2.2 \mathrm{mmol} / \mathrm{l}$ or the presence of glucose or protein in urine.
We estimated that we needed twenty-five subjects per group to detect a change of 1.5 (SD 1.9) kg body weight (our primary outcome measure). This sample size calculation was based on data collected by Parnell \& Reimer, which had a similar design and setting as the present study ${ }^{(28)}$. Furthermore, an $80 \%$ power and $\alpha=0.05$ were taken into account. Because of an expected dropout rate of $20 \%$, we aimed to include sixty participants in total. The participants were pair-matched according to sex, age, smoking status and BMI and sub sequently randomly allocated to the intervention group (bar with oligofructose (OF-Bar)) and the control group (bar without oligofructose (Co-Bar)) by an independent researcher. The study was triple-blind - that is participants and researchers were blind to the treatment during data collection and data analyses. At completion of the study, the subjects indicated in a questionnaire whether they knew which type of bar they had received. This was in order to check blinding, and after that they were debriefed. The study was performed according to the guidelines laid down in the Declaration of Helsinki, and all procedures involving human subjects were approved by the medical research ethics committee of Wageningen University (ABR 45006.081.13). Written informed consent was obtained and subjects received monetary compensation. This trial was registered at www.trialregister.nl as NTR 4075.

\section{Study design}

The present triple-blind, parallel, randomised controlled 12-week intervention study was carried out between September 2013 and January 2014 at the Division of Human Nutrition, Wageningen University. All participants were asked to consume a bar twice a day replacing their normal snack for 12 weeks. Throughout the study, participants lived at home, and purchased and prepared their own meals except on the 3 study days at baseline, weeks 6 and 12. Participants were provided with the following instructions: 'replace a snack you normally eat for the bar (comparable in energy with the bar) in the morning and in the afternoon and keep a normal activity pattern. Furthermore, consume the bars together with a glass of water, the first bar 30-90 min before lunch and the other bar 30-90 min before dinner'. In a meeting before the start of the study, the participants received examples of common snacks that could be replaced by the granola bar. The study was designed to examine the effects of oligofructose independent of any other diet or exercise regimen. Therefore, participants were asked to maintain their regular lifestyle, to eat until comfortably full and not to consciously try to lose or gain weight during the study.

\section{Screening procedures}

After receiving oral and written information and signing the consent form, ninety-one participants completed a health and lifestyle questionnaire to determine eligibility (Fig. 1). This among others included the Dutch Eating Behaviour Questionnaire $^{(31)}$ and a FFQ aimed to measure habitual fibre and EI. Next, the participants joined a screening test in fasting state. This test included a measurement of height and body weight wearing light clothes in order to check whether the participants had the required BMI. In addition, blood was drawn to check 


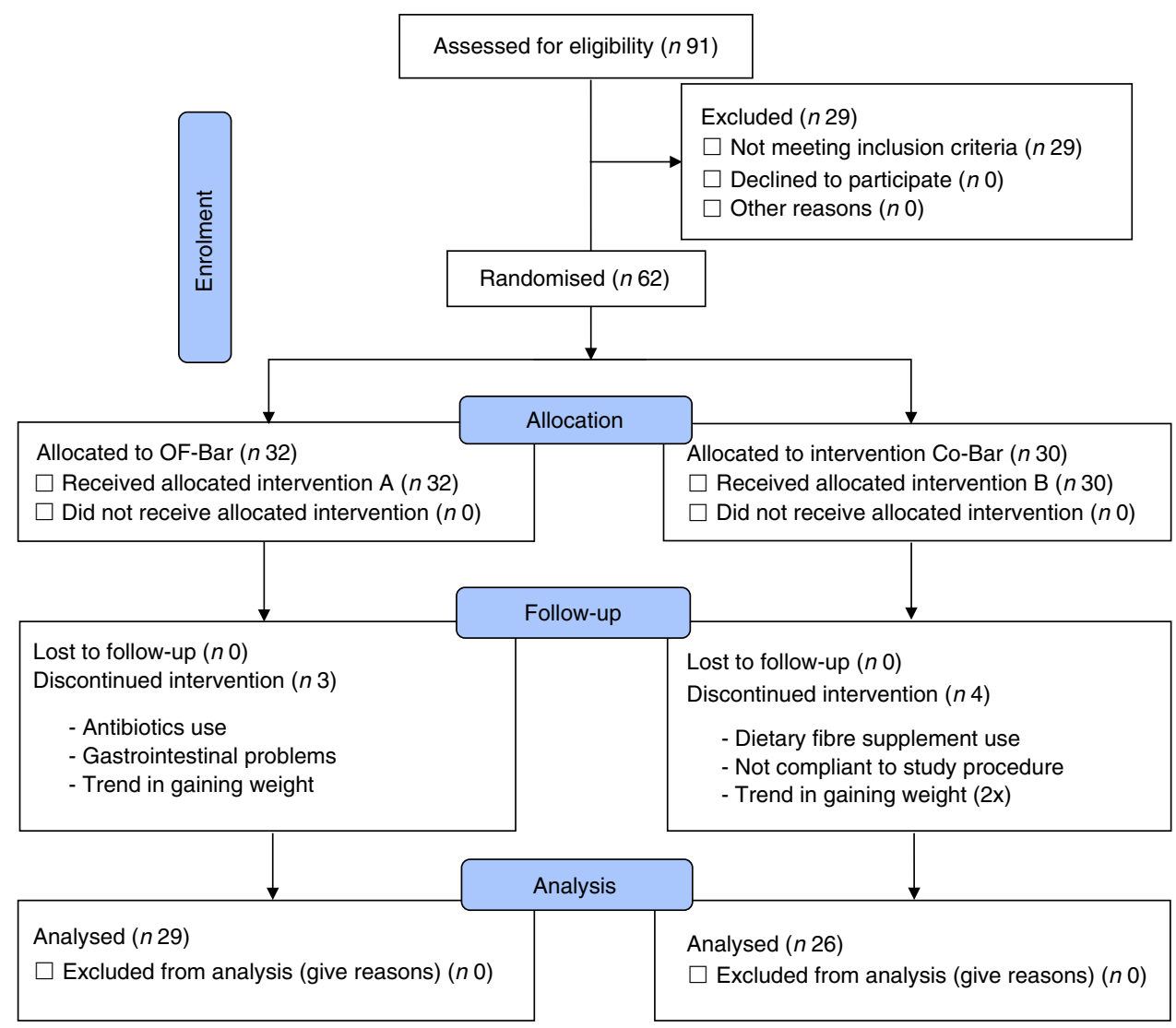

Fig. 1. Consolidated Standards of Reporting Trials (CONSORT) 2010 flow diagram of the inclusion of subjects in the intervention study. OF-Bar, oligofructose granola bar; Co-Bar, control granola bar.

fasting glucose, total cholesterol and TAG concentrations. Subsequently, urine was tested for glucose and proteins using a test strip.

\section{Study procedures}

For all test days, subjects had to arrive in a fasting state, which means that the participants were not allowed to eat after 21.00 hours and not to drink after 23.00 hours the evening before the test day. Each test day the participants arrived at the same time at the University between 07.15 and 10.00 hours. On the full test days (at baseline, weeks 6 and 12), the participants were invited for anthropometric measurements and their spontaneous food intake was measured during $24 \mathrm{~h}$. After weeks 1, 3 and 9, participants had a short test day to check whether the subject suffered from any discomfort and whether he or she succeeded in replacing his/her snacks by the granola bar by measuring body weight and waist circumference. During the first 3 weeks, weight gain was monitored; if subjects were systematically gaining over $0.2 \mathrm{~kg}$ of body weight/week, this was seen as an indication of not being able to equally replace their snacks. They were then contacted by our dietitian to give personalised advice to replace snacks equivalent in energy. In total, the dietitian contacted fifteen participants: nine in the OF-Bar group and six in the Co-Bar group. In week 6, these subjects were monitored again. At that moment, for ethical reasons, we excluded one participant in the OF-Bar group and two in the Co-Bar group who gained $>1.2 \mathrm{~kg}$ in the first 6 weeks.
Physical activity monitoring during the test day. Participants recorded their physical activity at baseline and after weeks 6 and 12 , in order to monitor maintenance of the physical activity level throughout the study. They used a 1-d retrospective questionnaire with fourteen categories ${ }^{(32,33)}$.

\section{Test product and intervention}

The OF-Bar group received two chocolate chip, chewy granola bars in which $8 \mathrm{~g}$ of oligofructose (Frutalose ${ }^{\circledR}$ L92; Sensus, containing $92 \%$ oligofructose and $8 \%$ monosaccharides and disaccharides on a dry weight basis) was added per bar - that is a total of $16 \mathrm{~g}$ of oligofructose on a daily basis. The 16-g dose was chosen based on a previous study, which suggested a positive impact on EI and appetite, and was well tolerated ${ }^{(21)}$. The Co-Bar group received a control chocolate chip, chewy granola bar without oligofructose. In the Co-Bar, oligofructose was substituted with sugars, mainly coming from high-maltose maize syrup, maize syrup and starch (from crisp rice). The amount of maltodextrin in the Co-Bar was $1.7 \%$ (dwb) compared with $0 \%$ (dwb) in the OF-Bar. The bars were cold-formed and not subjected to temperatures above $65^{\circ} \mathrm{C}$. The bars were packaged per portion in identical opaque packages to ensure the double-blind status of the study. Moreover, the bars were coded by an independent colleague and investigators, and participants did not know who received which type of bar. Compositions of both bars are presented in Table 1. The bars were kindly provided by Sensus, Roosendaal, The Netherlands. 
Table 1. Weight and nutrient composition of the isoenergetic granola bars per bar

(Mean values and standard deviations)

\begin{tabular}{|c|c|c|}
\hline & Co-Bar & OF-Bar \\
\hline \multicolumn{3}{|l|}{ Weight $(\mathrm{g})^{*}$} \\
\hline Mean & 27 & 32 \\
\hline $\mathrm{SD}$ & 2 & 2 \\
\hline Energy $(\mathrm{kJ}) \dagger$ & 417 & 411 \\
\hline Energy (kcal)‡ & 99 & 98 \\
\hline Protein $(\mathrm{g}) \S$ & 1.2 & 1.2 \\
\hline Carbohydrates (g)\| & $19 \cdot 8$ & $25 \cdot 5$ \\
\hline OF $(g) \|$ & 0.2 & $10 \cdot 1$ \\
\hline Fat $(g) \S$ & 1.7 & $1 \cdot 3$ \\
\hline
\end{tabular}

Co-Bar, control granola bar; OF-Bar, oligofructose granola bar; OF; oligofructose.

* Forty granola bars.

† Calculated as protein $17 \mathrm{~kJ}$, carbohydrates $17 \mathrm{~kJ}$, OF $6 \mathrm{~kJ}$, fat $37 \mathrm{~kJ}$

Calculated as protein $17 \mathrm{~kJ}$ (4 kcal), carbohydrates $17 \mathrm{~kJ}$ (4 kcal), OF $6.3 \mathrm{~kJ}(1.5 \mathrm{kcal})$, fat 37.7 (9 kcal).

$\S$ Determined at the Division of Human Nutrition.

II Determined at the Department of Food Chemistry. Calculated based on average degree of polymerisation $=4$.

During the 1st week, the participants ate one bar per day in order to prevent gastrointestinal discomfort caused by a sudden increase in dietary fibre consumption. The bars - including some spare ones - were provided to the participants for the upcoming period at the end of every meeting at the University. Subjects were instructed to bring leftovers back to University at the next meeting, so that we could count their compliance. Furthermore, participants were asked to write down the time at which they ate the bars in a daily journal.

\section{Outcome variables}

Body weight and body composition. Height was measured once during the screening visit; it was measured to the nearest $\mathrm{mm}$, with the participant standing erect on bare feet and the head in the 'Frankfurt plane' using a wall-mounted stadiometer. Body weight was measured to the nearest $0.1 \mathrm{~kg}$ using calibrated electronic weighing scales (Seca 877 and Tanita ${ }^{\circledR}$-scale attached to the BodPod; Life Measurement Inc.). Waist circumference was measured to the nearest $0.25 \mathrm{~cm}$ using a measuring tape at the mid-point between the ribs and the hips. At the test days (at baseline and after weeks 1, 3, 6, 9 and 12), participants were assessed on body weight and waist circumference in the fasting state, after relieving themselves, wearing the same swim suit at every visit.

At the test days (baseline, weeks 6 and 12), body composition was non-invasively determined using air-displacement plethysmography by using the BodPod device according to the manufacturer's guidelines (Life Measurement Inc. of Cosmed). A two-step calibration was carried out before each measurement. All participants wore a tight-fitting swim suit and hat. Body weight was measured, and then two repeated body volume measurements were performed, averaged and corrected for the predicted body surface area (BSA) and predicted thoracic gas volume, to obtain the body density. To convert body density to percentage body fat, Brozek's ${ }^{(34,35)}$ two-compartment model was used. Fat-free mass was calculated as the difference between each participant's body weight and body FM. Body density is dependent on body weight and body volume (body density=body weight/body volume).
Body volume $=$ raw volume + BSA artifact $+0 \cdot 4 \times$ Lung volume. BSA was calculated as BSA $=71.84 \times \mathrm{BW}^{0.425} \times$ height $^{0.725}$. BSA artefact was as follows: BSA $\times k$ (a constant which is $\left.4.67 \times 10^{-5}\right)^{(36)}$. Then, the Brozek's equation, where \% FM $=(4 \cdot 57 /$ body density $-4 \cdot 142)$ $\times 100$, was used.

Food intake during the test day. To measure spontaneous 24-h food intake, all meals were served ad libitum during the test day. After the anthropometric measurements, participants received day- 1 breakfast in buffet style at the University; subsequently, they received a bag with lunch and snacks, which they were allowed to eat as much they wanted at home or at work until dinner. These bags also contained the bars, which were mandatory (at baseline, all participants received two control bars). Between 17:00 and 18:00, a pasta meal was served in portions of $200 \%$ of the amount they would eat according to their assumed EI according to the Harris-Benedict formula. For the evening, participants received a bag with snacks. The subsequent morning participants had the day-2 buffet breakfast at the University. Participants were asked not to eat or drink something outside the provided foods, except for coffee, tea and water (without sugar or milk). The food items, which participants could choose, were foods Dutch people habitually eat. For a complete list of all foods, see the online Supplementary Appendix A. Food was offered in excess to minimise external food intake cues. Food intake was calculated by weighing all food and drinks before serving and weighing leftovers. Energy and macronutrient intake was estimated using the Dutch Food Composition Database.

Subjective appetite during the test day. Subjective hunger, fullness, prospective food consumption, desire to eat and thirst were registered every hour on eleven-point scales with an anchor on the left side 'not at all' and on the right side 'very much'(37). Participants completed these questionnaires before they ate breakfast, then after breakfast and then every hour that participants were awake. In addition, before and after lunch and before and after dinner participants completed these questionnaires. The questionnaire was provided in the form of a booklet for each test day. For the analysis, the mean values of the appetite parameters and the mean time were divided to calculate weighted hunger, fullness, desire to eat, prospective food consumption and thirst.

Weekly appetite questionnaires, liking of the bar and tolerance. Every week participants completed 100-mm visual analogue scale (VAS) questionnaires during which they reflected on their appetite feelings of the last week. In addition, liking of the bars and tolerance, that is bloating, regurgitation, flatulence, nausea and looser stools, were measured by $100 \mathrm{~mm}$ VAS, anchored with "not at all" $(0 \mathrm{~mm})$ to "very much" $(100 \mathrm{~mm})$.

\section{Statistical analyses}

Results are expressed as means and standard deviations, unless stated otherwise. Variables were visually checked for normal distribution; all data were normally distributed. Baseline differences between intervention groups were tested by means of an unpaired Student's $t$ test. Data were analysed by means of 
repeated mixed-model ANOVA (PROC MIXED). Best fitted covariance structures were used per model. Models included treatment, time and treatment $\times$ time interaction. Post hoc tests were performed with least significant difference test. The level of significance was set at $P<0 \cdot 05$. All data were analysed using SAS 9.3 (SAS Institute).

Unblinding of the trial occurred in two steps. Study investigators performed the primary analyses while being blind to the treatments, which means that they knew which participants were in a group together. After these analyses the treatments were fully unblinded.

\section{Results \\ Subjects}

A total of sixty-two suitable and willing participants were enrolled in the study, among whom forty-eight started in September and forty-eight in November 2013. Finally, fifty-five completed the total study (OF-Bar: $n$ 29, Co-Bar: $n$ 26). Characteristics of fifty-five participants whose data were analysed are presented in Table 2. In total, three participants in the OF-Bar group and four participants in the Co-Bar group dropped out. There were no significant differences between the groups in any of the baseline characteristics. During the study the level of physical activity did not differ between both groups (Co-Bar baseline: 1.56 (SD 0.21); week 12: 1.51 (SD 0.22); and OF-Bar baseline: 1.60 (SD 0.18); week 12: 1.56 (sD 0.21) physical activity level). Counting the returned bars showed that on average $98 \%$ of the 162 bars that needed to be eaten were eaten and all subjects reported that they consumed over $90 \%$ of the bars during the intervention period. Eventually, to the question 'which bar do you think you had?', 30.9 and $12.7 \%$ of the subjects for the control bar and oligofructose bar, respectively, were correct in their answer; $30.9 \%$ received the oligofructose bar and thought to be in the Co-Bar group; $21.8 \%$ received

Table 2. Baseline characteristics of study participants (Mean values and standard deviations)

\begin{tabular}{|c|c|c|c|c|}
\hline & \multicolumn{2}{|c|}{ Co-Bar (n 26) } & \multicolumn{2}{|c|}{ OF-Bar (n 29) } \\
\hline & Mean & SD & Mean & SD \\
\hline Female/male $(n / n)$ & \multicolumn{2}{|c|}{$18 / 8$} & \multicolumn{2}{|c|}{$18 / 11$} \\
\hline Age $\left(\right.$ years) ${ }^{*}$ & 39 & 12 & 42 & 12 \\
\hline Height $(m)^{\star}$ & 1.75 & 0.10 & $1 \cdot 75$ & $0 \cdot 10$ \\
\hline Body weight $(\mathrm{kg})^{*}$ & $90 \cdot 2$ & $12 \cdot 6$ & $90 \cdot 9$ & $11 \cdot 3$ \\
\hline BMI $\left(\mathrm{kg} / \mathrm{m}^{2}\right)^{\star}$ & $29 \cdot 7$ & $2 \cdot 8$ & $29 \cdot 7$ & 2.4 \\
\hline Waist circumference $(\mathrm{cm})^{\star}$ & $99 \cdot 0$ & $8 \cdot 2$ & $97 \cdot 6$ & $8 \cdot 4$ \\
\hline Body fat $(\%)^{*} \dagger$ & $38 \cdot 6$ & $7 \cdot 4$ & 37.5 & $8 \cdot 1$ \\
\hline Body fat $(\mathrm{kg})^{*} \dagger$ & $35 \cdot 0$ & $9 \cdot 7$ & $34 \cdot 1$ & $8 \cdot 1$ \\
\hline Physical activity level* $\ddagger$ & 1.6 & $0 \cdot 2$ & 1.6 & $0 \cdot 2$ \\
\hline Habitual EI $(\mathrm{MJ})^{\star} \S$ & $9 \cdot 0$ & 1.9 & $8 \cdot 7$ & 2.4 \\
\hline Habitual fibre intake $(g)^{\star} \S$ & $22 \cdot 0$ & $5 \cdot 3$ & $22 \cdot 2$ & $6 \cdot 9$ \\
\hline Restraint eating score $\|^{*}$ & 2.92 & 0.86 & $2 \cdot 82$ & 0.49 \\
\hline
\end{tabular}

Co-Bar, control group; OF-Bar, oligofructose group; El, energy intake.

* Independent samples $t$ test showed no statistical significant difference between groups.

† Measured by BodPod (Life Measurement Inc. of Cosmed).

$\mp$ Calculated from a 24-h physical activity recorded in the diary ${ }^{(32,33)}$

$\S$ Habitual intake estimated by means of a FFQ completed before the start of the study.

II Measured with the Dutch Eating Behaviour Questionnaire ${ }^{(31)}$, scores $1-5,1$ is low restrained and 5 is high restrained. the control bar and thought to be in the OF-Bar group; and $3.6 \%$ had no idea, while they were in the Co-Bar group.

\section{Body weight and body composition}

OF-Bar consumption did not result in beneficial changes in body weight change and body composition compared with the Co-Bar (all time $\times$ treatment effects; $P>0.05$ (Table 3)). Over time, body weight did not change in both groups, whereas waist circumference decreased (time effect: $P<0 \cdot 0001$ ). Body composition measured by air-displacement plethysmography showed that percentage $(P=0.03)$ and absolute lean body mass $(P=0.001)$ decreased in both groups. Percentage FM increased in both groups $(P=0.03)$, whereas absolute FM did not change $(P=0 \cdot 17)$.

\section{Food intake during the test day}

Food intake was not different at baseline, except for the baseline values of the day-2 breakfast, which were significantly lower for the OF-Bar group (Co-Bar: $2 \cdot 0(\mathrm{SD} 0 \cdot 7) \mathrm{MJ} v$. OF-Bar: 1.6 (sD 0.4$) \mathrm{MJ} ; P=0.01$ ). The addition of oligofructose did not significantly affect total EI. At baseline, EI was 9882 (SD 2541) and 9334 (SD 1983) kJ with the Co-Bar and OF-Bar group, respectively. After 6 weeks, EI increased in the Co-Bar group to 10089 (sD 2902) kJ and reduced in the OF-Bar group to 8965 (sD 2252) kJ (time $\times$ treatment $P=0 \cdot 41$ ). There were no significant differences for any meal or snack moment (Table 4). The day-1 breakfast showed a significant increase in EI over time for both groups $(P=0.03)$ (Table 4). EI at lunch did not change. At dinner, EI showed a downward trend over time for both groups $(P=0 \cdot 07)$. In both groups, the evening snack intake tended to decrease at week $6(-0 \cdot 1 \mathrm{MJ}$; NS), but significantly increased in week 12 compared with week 6 $(+0 \cdot 2 \mathrm{MJ} ; P=0 \cdot 01)$.

\section{Subjective appetite during the test day}

At baseline, both groups were not different from each other for the five scales for weighted average appetite/satiety outcomes (all $P>0.05$ ). We found no significant effect of oligofructose supplementation on the weighted average values for hunger, fullness, prospective consumption, desire to eat or thirst (Table 5). Only hunger tended to be lower in the OF-Bar group at baseline $(-0.4 ; P=0.09)$.

\section{Weekly appetite questionnaires}

Weekly hunger, fullness, desire to eat, prospective food consumption and thirst ratings were not significantly different at baseline for the OF-Bar and Co-Bar group. As shown in Fig. 2, participants of the OF-Bar group reported to have significantly less overall hunger and thirst (time $\times$ treatment $P=0.044$ respectively $P=0.003$ ) compared with the Co-Bar group. Moreover, ratings for prospective consumption were lower for the OF-Bar group compared with the Co-Bar group (time $\times$ treatment $P=0.03$ ). A trend was seen that participants consuming oligofructose tended to have a lower desire to eat compared with the Co-Bar group (time $\times$ treatment $P=0.097$ ). There was no difference in fullness (time $\times$ treatment $P=0.23$ ). 
Table 3. Body weight, waist circumference and body composition during the study

(Mean values and standard deviations)

\begin{tabular}{|c|c|c|c|c|c|c|c|c|c|c|c|c|c|c|c|}
\hline \multirow[b]{3}{*}{ Treatments } & \multicolumn{12}{|c|}{ Time (weeks) } & & & \\
\hline & \multicolumn{2}{|c|}{0} & \multicolumn{2}{|c|}{1} & \multicolumn{2}{|c|}{3} & \multicolumn{2}{|c|}{6} & \multicolumn{2}{|c|}{9} & \multicolumn{2}{|c|}{12} & \multicolumn{3}{|c|}{ ANOVA mixed model $(P)$} \\
\hline & Mean & SD & Mean & SD & Mean & SD & Mean & SD & Mean & SD & Mean & SD & Treatment & Time (weeks) & Treatment $\times$ time \\
\hline \multicolumn{16}{|c|}{ Body weight (kg) } \\
\hline Co-Bar & $90 \cdot 2$ & $12 \cdot 6$ & $90 \cdot 2$ & $12 \cdot 7$ & $90 \cdot 1$ & $13 \cdot 1$ & $90 \cdot 5$ & 12.5 & $90 \cdot 5$ & $12 \cdot 5$ & $90 \cdot 6$ & $12 \cdot 6$ & 0.89 & 0.23 & 0.54 \\
\hline OF-Bar & $90 \cdot 9$ & $11 \cdot 3$ & $90 \cdot 9$ & $11 \cdot 1$ & $90 \cdot 6$ & 11.4 & $90 \cdot 7$ & $11 \cdot 3$ & $90 \cdot 7$ & $11 \cdot 2$ & $90 \cdot 7$ & $11 \cdot 3$ & & & \\
\hline \multicolumn{16}{|l|}{ Waist (cm) } \\
\hline Co-Bar & $99 \cdot 0^{\mathrm{a}}$ & $8 \cdot 2$ & $99 \cdot 1^{a}$ & $8 \cdot 3$ & $98 \cdot 9^{a}$ & 8.7 & $96 \cdot 6^{\mathrm{b}}$ & 8.6 & $98 \cdot 5^{a}$ & $8 \cdot 1$ & $96 \cdot 6^{\mathrm{b}}$ & 8.4 & 0.62 & $<0.0001$ & 0.70 \\
\hline OF-Bar & $97 \cdot 6^{a}$ & 8.4 & $98 \cdot 0^{a}$ & $7 \cdot 6$ & $97 \cdot 4^{\mathrm{a}}$ & 8.0 & $96 \cdot 3^{b}$ & $8 \cdot 2$ & $97 \cdot 1^{a}$ & 8.5 & $95 \cdot 6^{\mathrm{b}}$ & 8.8 & & & \\
\hline \multicolumn{16}{|c|}{ Fat mass $(\mathrm{kg})$} \\
\hline Co-Bar & $35 \cdot 0$ & $9 \cdot 7$ & & & & & $35 \cdot 2$ & 9.9 & & & 35.6 & $9 \cdot 8$ & 0.67 & 0.17 & 0.37 \\
\hline OF-Bar & $34 \cdot 1$ & $8 \cdot 1$ & & & & & 34.4 & 7.9 & & & $34 \cdot 3$ & $7 \cdot 6$ & & & \\
\hline \multicolumn{16}{|c|}{ Lean mass $(\mathrm{kg})$} \\
\hline Co-Bar & $55 \cdot 3^{a}$ & $9 \cdot 1$ & & & & & $54 \cdot 9^{b}$ & $9 \cdot 0$ & & & $55 \cdot 1^{a}$ & $9 \cdot 3$ & 0.59 & $<0.01$ & 0.63 \\
\hline OF-Bar & $56 \cdot 9^{a}$ & $10 \cdot 6$ & & & & & $56 \cdot 3^{b}$ & $10 \cdot 6$ & & & $56 \cdot 4^{a}$ & 10.5 & & & \\
\hline \multicolumn{16}{|l|}{ Body fat $\%$} \\
\hline Co-Bar & $38.6^{a}$ & $7 \cdot 4$ & & & & & $38.9^{b}$ & $7 \cdot 3$ & & & $39 \cdot 1^{b}$ & $7 \cdot 4$ & 0.63 & 0.03 & 0.42 \\
\hline OF-Bar & $37 \cdot 5^{\mathrm{a}}$ & $8 \cdot 1$ & & & & & $38 \cdot 0^{\mathrm{b}}$ & $8 \cdot 0$ & & & $37.9^{b}$ & $7 \cdot 7$ & & & \\
\hline
\end{tabular}

Co-Bar, control granola bar; OF-Bar, oligofructose granola bar.

${ }^{\mathrm{a}, \mathrm{b}}$ Mean values with unlike superscript letters were significantly different $(P<0.05)$.

Table 4. Dietary energy intake on an ad libitum test day

(Mean values and standard deviations)

\begin{tabular}{|c|c|c|c|c|c|c|c|c|c|}
\hline & \multicolumn{2}{|c|}{ Week 0} & \multicolumn{2}{|c|}{ Week 6} & \multicolumn{2}{|c|}{ Week 12} & \multicolumn{3}{|c|}{ ANOVA mixed model $(P)$} \\
\hline & Mean & $\mathrm{SD}$ & Mean & $\mathrm{SD}$ & Mean & $\mathrm{SD}$ & Treatment & Time (weeks) & Treatment $\times$ time \\
\hline \multicolumn{10}{|c|}{ Total energy intake (kJ) } \\
\hline Co-Bar & 9882 & 2541 & 10089 & 2902 & 10133 & 2538 & $0 \cdot 18$ & 0.68 & 0.41 \\
\hline OF-Bar & 9334 & 1983 & 8965 & 2252 & 9299 & 2408 & & & \\
\hline \multicolumn{10}{|c|}{ El day-1 breakfast (kJ) } \\
\hline Co-Bar & $1842^{\mathrm{a}}$ & 767 & $1993^{b}$ & 854 & $1996^{b}$ & 771 & 0.17 & 0.03 & 0.98 \\
\hline OF-Bar & $1602^{a}$ & 520 & $1738^{b}$ & 647 & $1764^{b}$ & 581 & & & \\
\hline \multicolumn{10}{|c|}{ El lunch and snack bag (kJ) } \\
\hline Co-Bar & 3429 & 1060 & 3707 & 1140 & 3543 & 1089 & $0 \cdot 21$ & 0.78 & 0.52 \\
\hline OF-Bar & 3253 & 1160 & 3187 & 1320 & 3238 & 1250 & & & \\
\hline \multicolumn{10}{|c|}{ El dinner $(\mathrm{kJ})$} \\
\hline Co-Bar & 3279 & 1182 & 3205 & 1286 & 3237 & 1124 & 0.92 & 0.07 & 0.25 \\
\hline OF-Bar & 3461 & 755 & 3102 & 764 & 3086 & 714 & & & \\
\hline \multicolumn{10}{|c|}{ El evening snacks (kJ) } \\
\hline Co-Bar & $1332^{a, b}$ & 834 & $1183^{a}$ & 767 & $1357^{\mathrm{b}}$ & 882 & 0.22 & 0.04 & 0.63 \\
\hline OF-Bar & $1017^{a, b}$ & 815 & $938^{a}$ & 633 & $1211^{b}$ & 801 & & & \\
\hline \multicolumn{10}{|c|}{ El day-2 breakfast (kJ) } \\
\hline Co-Bar & 2006 & 727 & 1838 & 601 & 1995 & 748 & 0.06 & 0.45 & 0.12 \\
\hline OF-Bar & 1584 & 447 & 1702 & 581 & 1714 & 586 & & & \\
\hline
\end{tabular}

Co-Bar, control granola bar; OF-Bar, oligofructose granola bar, El, energy intake.

a,b Mean values with unlike superscript letters were significantly different $(P<0.05)$.

\section{Liking of the bars and tolerance}

At baseline, everybody received the control bar, so they rated the control bar for liking at baseline.

Fig. 3 shows that the liking of the oligofructose bar is quickly reduced compared with the control bar $(P=0.03)$. Moreover, this figure also shows a decline in liking of both bars $(P=0.02)$.

Flatulence, bloating, looser stools, regurgitation and nausea were not significantly different at baseline for the OF-Bar and Co-Bar groups. In the first 2 weeks, the OF-Bar group perceived significantly more flatulence and bloating than at baseline. Notably from week 7 , both groups noted no differences for tolerance scores between the Co-Bar and OF-Bar (Fig. 4).

\section{Discussion}

The results showed that 12 -week replacement of snacks twice a day with granola bars enriched with $8 \mathrm{~g}$ of oligofructose had no significant impact on weight management outcome measures, such as body weight, body composition, EI and acute appetite in a group of overweight or obese adults. However, weekly appetite feelings seemed to be lower in the OF-Bar group compared with the Co-Bar group.

The results of the present study on body weight are in contrast to the findings of other long-term randomised controlled trials. In the present study, the non-significant difference in body weight was +0.4 and $-0.2 \mathrm{~kg}$ for the Co-Bar and 
Table 5. Weighted average score of appetite during the test day (Mean values and standard deviations)

\begin{tabular}{|c|c|c|c|c|c|c|c|c|c|}
\hline & \multicolumn{2}{|c|}{ Week 0} & \multicolumn{2}{|c|}{ Week 6} & \multicolumn{2}{|c|}{ Week 12} & \multicolumn{3}{|c|}{ ANOVA mixed model $(P)$} \\
\hline & Mean & SD & Mean & SD & Mean & SD & Treatment & Time (week) & Treatment $\times$ time \\
\hline \multicolumn{10}{|l|}{ Hunger } \\
\hline Co-Bar & 3.9 & 1.2 & 4.1 & 1.0 & 4.2 & 1.0 & \multirow[t]{2}{*}{0.09} & \multirow[t]{2}{*}{0.59} & \multirow[t]{2}{*}{0.46} \\
\hline OF-Bar & $3 \cdot 7$ & 1.0 & $3 \cdot 7$ & 1.0 & 3.7 & $1 \cdot 1$ & & & \\
\hline \multicolumn{10}{|l|}{ Fullness } \\
\hline Co-Bar & $6 \cdot 9$ & 1.4 & $7 \cdot 0$ & $1 \cdot 1$ & $7 \cdot 0$ & $1 \cdot 2$ & \multirow[t]{2}{*}{0.72} & \multirow[t]{2}{*}{$0 \cdot 10$} & \multirow[t]{2}{*}{0.23} \\
\hline OF-Bar & 6.5 & 1.4 & $7 \cdot 0$ & 1.3 & $7 \cdot 1$ & 1.5 & & & \\
\hline \multicolumn{10}{|c|}{ Desire to eat } \\
\hline Co-Bar & 4.1 & $1 \cdot 2$ & 4.3 & 1.0 & 4.5 & 1.2 & \multirow[t]{2}{*}{0.23} & \multirow[t]{2}{*}{0.91} & \multirow[t]{2}{*}{0.24} \\
\hline OF-Bar & 4.1 & $1 \cdot 2$ & $4 \cdot 0$ & 1.2 & $3 \cdot 9$ & $1 \cdot 1$ & & & \\
\hline \multicolumn{10}{|c|}{ Prospective consumption } \\
\hline Co-Bar & 4.1 & $1 \cdot 1$ & $4 \cdot 2$ & $1 \cdot 1$ & 4.4 & 1.1 & \multirow[t]{2}{*}{0.35} & \multirow[t]{2}{*}{0.48} & \multirow[t]{2}{*}{0.19} \\
\hline OF-Bar & $4 \cdot 1$ & 1.4 & 3.9 & 1.3 & 3.9 & 1.2 & & & \\
\hline \multicolumn{10}{|l|}{ Thirst } \\
\hline Co-Bar & $5 \cdot 0$ & 1.5 & 4.9 & 1.5 & $5 \cdot 1$ & 1.5 & \multirow[t]{2}{*}{0.23} & \multirow[t]{2}{*}{0.91} & \multirow[t]{2}{*}{0.24} \\
\hline OF-Bar & 4.7 & 1.8 & 4.6 & $2 \cdot 1$ & $4 \cdot 3$ & 1.9 & & & \\
\hline
\end{tabular}

Co-Bar, control granola bar; OF-Bar, oligofructose granola bar.

(a)

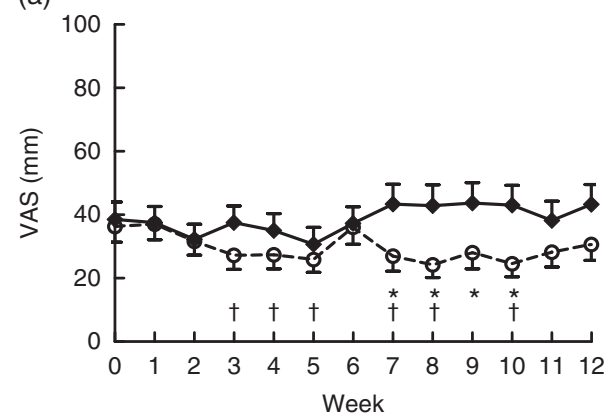

(c)

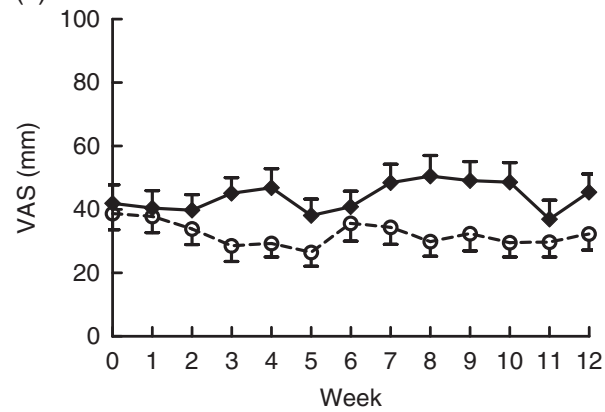

(b)

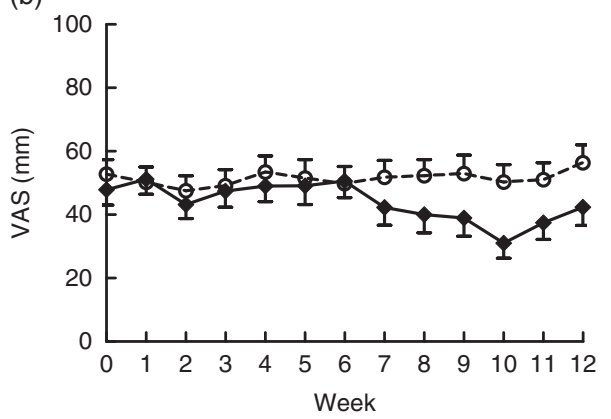

(d)

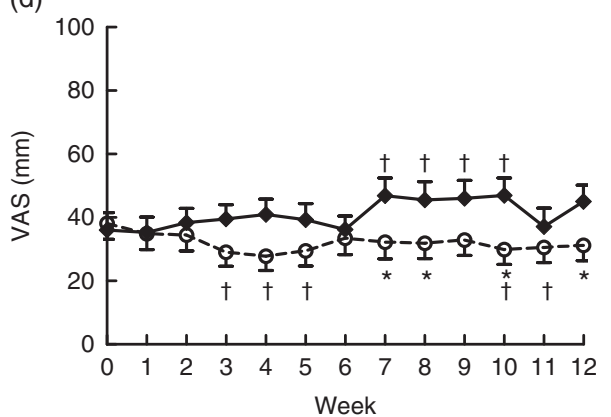

(e)

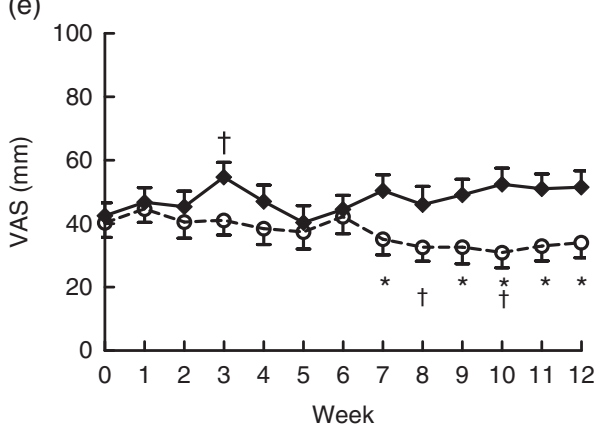

Fig. 2. Effects of oligofructose bars (OF-Bar; $8 \mathrm{~g}$ oligofructose twice daily) or control bars (Co-Bar) as replacement for a morning and mid-day snack for 12 weeks on feelings of hunger (a), fullness (b), desire to eat (c), prospective food consumption (d) and thirst (e) measured weekly on a visual analogue scale (VAS). Baseline values are not different. Values are means with their standard errors. * Mean value OF-Bar was significantly different from control at the same week $(P<0.05)$. $\dagger$ Mean value was significantly different from that at baseline (week 0$)$ for the same group $(P<0.05)$. 
OF-Bar group, respectively, after 12 weeks. In comparison, another study using $21 \mathrm{~g} / \mathrm{d}$ oligofructose reported a significant change in body weight of +0.5 and $-1.0 \mathrm{~kg}$ in the control group and the oligofructose group after 12 weeks of supplementa$\operatorname{tion}^{(28)}$. On the other hand, another study with a higher dose of $30 \mathrm{~g} / \mathrm{d}$ oligofructose did not affect body weight in overweight and obese subjects following 8 weeks of supplementation ${ }^{(29)}$. However, when inulin was supplemented as $30 \mathrm{~g} / \mathrm{d}$ during and

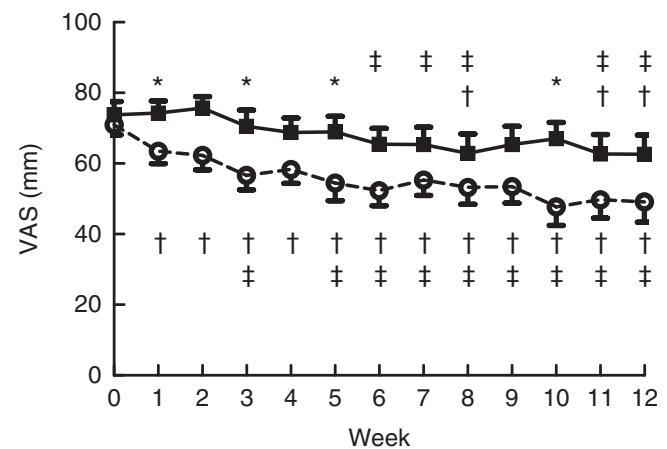

Fig. 3. Liking of the bars during the intervention. Main effects: treatment (Tr), $P=0.03$; time (Ti), $P=0.02 ; \operatorname{Tr} \times \mathrm{Ti}, P=0.03$. Values are means with their standard errors. - - , Control granola bar (Co-Bar); - $\bigcirc-$, oligofructose granola bar (OF-Bar); VAS, visual analogue scale. * Mean value was significantly different from OF-Bar at the same week $(P<0.05)$. The mean difference between treatments was $12 \mathrm{~mm}$. † Mean value was significantly different from that at baseline (week 0 ) for the same group $(P<0.05)$. $\ddagger$ Mean value was significantly different from that at week 1 for the same group $(P<0.05)$. after an active weight loss phase, body weight and FM were significantly reduced ${ }^{(30)}$. The instruction in the present study was only to replace a snack, so not to actively lose weight, which may have diminished the effect on body weight reduction.

Despite the instruction to replace a snack that one typically consumes with the snack bar (comparable in energy with the bar) in the morning and in the afternoon, maybe the subjects were not able to replace their daily snack as instructed. If subjects were not able to adequately replace their snacks during these 12 weeks, they could gain approximately $2 \mathrm{~kg}$ by eating the bars on top of their diet. As these subjects already were overweight, substantial weight gain has to be avoided during the study. Therefore, subjects whose weight increased during the first 3 weeks were phoned by the dietitian in order to help them find a suitable snack from their regular diet that could be replaced with the bar. Of the fifteen subjects that our dietitian called, nine were in the OF-Bar group and six in the Co-Bar group. In case subjects still gained weight after 6 weeks, they were omitted from the study; this occurred with three subjects (one subject in the OF-Bar group and two subjects in the Co-Bar group).

The non-significant changes in body weight, body composition and EI may have been influenced by the relatively small sample size or insufficient power to detect slight differences between treatments. Our sample size estimation was based on a study using a higher dose of oligofructose for which we did not correct $^{(21)}$. However, a similar study of Guess et al. ${ }^{(30)}$ with only thirty-nine subjects did find positive results consuming $30 \mathrm{~g} / \mathrm{d}$
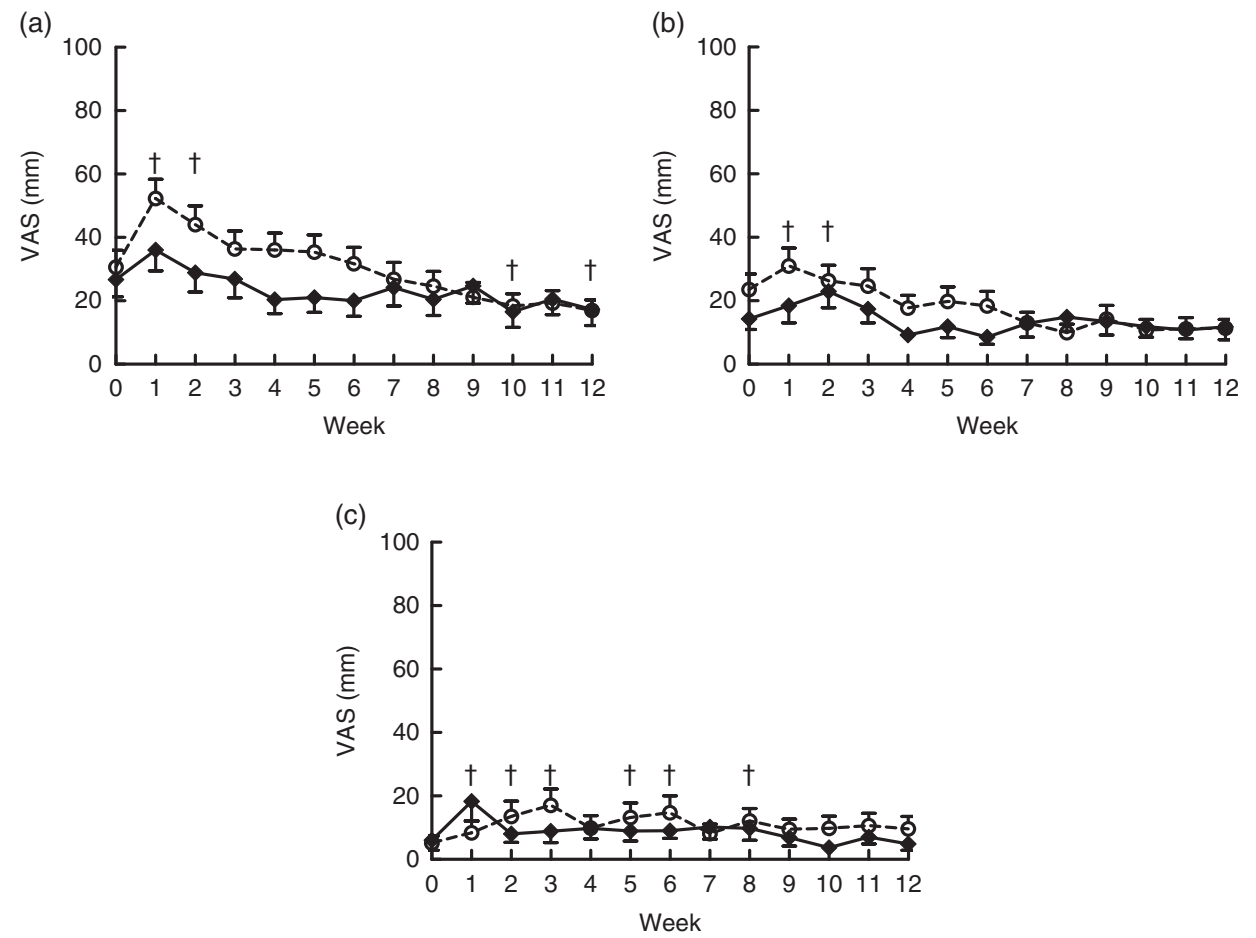

Fig. 4. Effects of oligofructose bars (OF-Bar; $8 \mathrm{~g}$ oligofructose per bar, twice daily) or control bars (Co-Bar) as replacement for a morning and mid-day snack for 12 weeks on feelings of flatulence (a), bloating (b) and looser stools (c) measured weekly on a visual analogue scale (VAS). Regurgitation and nausea were also measured, but these were low and not significantly different. Values are means with their standard errors. $†$ Mean value week was significantly different from that at baseline $(P<0.05)$. (a) Main effects: treatment $(\mathrm{Tr}), P=0.15$; time $(\mathrm{Ti}), P \leq 0.0001$; $\operatorname{Tr} \times \mathrm{Ti}, P=0.22$. Week 1 was significantly different compared with all other weeks $(P<0.05)$. (b) Main effects: treatment (Tr), $P=0.20$; time (Ti), $P=0.01 ; \operatorname{Tr} \times \mathrm{Ti}, P=0.29$. (c) Main effects: treatment $(\mathrm{Tr}), P=0.53 ;$ time (Ti), $P=0.045 ; \operatorname{Tr} \times \mathrm{Ti}, P=0.08$. 
inulin in the weight maintenance phase. The effect we found on body composition over time - that is decrease of lean body mass, waist circumference and an increase of percentage of FM - was similar in both intervention groups. The reduction of lean body mass, as this is mainly muscle mass, could have been caused by a decreased level of physical activity during the end of the calendar year ${ }^{(38)}$. However, no large changes in physical activity levels were reported during the intervention. Presumably, the measurement days were not reflecting an average day of the intervention period. Another cause for the reduction of lean body mass may be an alteration in diet composition; however, we did not assess diet composition.

During the intervention, we asked subjects to report their satiety feelings in two different ways. First, we asked them to fill out a satiety questionnaire during the test days; i.e. they rated their appetite every hour that they were awake with five different parameters. Second, every week subjects reported their satiety feelings of the last week in the study diary on similar parameters. Hunger scores during the test days tended to be lower in the OF-Bar group. However, the other parameters did not show such an effect. On the other hand, data from the weekly questionnaire substantiated these data; the OF-Bar group reported lower hunger, lower desire to eat and lower prospective consumption. Therefore, it might be that consuming additional oligofructose affected satiety feelings, but that filling out appetite ratings every hour is not an appropriate measure for this specific group of subjects. Weekly ratings filled out at home might give possibly more valid answers. This hypothesis is supported by the satiety feelings reported in week 6 , which show deviating results compared with the overall pattern of ratings during the 12 weeks of intervention. We speculate that coming to the research centre has an effect on these outcomes, which is visible in weekly hunger, prospective consumption, desire to eat and thirst. For the measurements during the test days, we used the Likert scales, which are less sensitive compared with a VAS. In addition, every participant had different timings of food consumption and could eat ad libitum, which could have affected the sensitivity of the hourly appetite questionnaire, because subjects were full after each eating occasion.

Similar to some other studies ${ }^{(23,29)}$, we did not find an effect on daily EI. The average EI of the subjects on the test days was relatively low, although with a large variation. During the intervention, subjects ate on average $9.6 \mathrm{MJ}$, which is about $81 \%$ of the energy needs that we estimated in the beginning of the intervention $(11 \cdot 8 \mathrm{MJ})$. Although this group was not very physically active, as indicated by their low physical activity score of $1 \cdot 5-1 \cdot 6$, this intake is very low. Considering their stable body weight, this may not reflect their normal intake. Otherwise, overweight and obese adults may have consumed less owing to socially desirable behaviour, as they know their eating behaviour was being observed ${ }^{(39)}$. We measured EI with an ad libitum method, as described in previous stu$\operatorname{dies}^{(37,40)}$, as it is well known that overweight subjects tend to underreport their intake by means of diaries ${ }^{(41,42)}$. We observed that subjects tended to eat more breakfast and evening snacks, and less dinner over time. This might indicate that they got bored with the dinner and compensated this with their evening snacks and breakfast. We observed a small mean decline in EI of 369 (sD 1490$) \mathrm{kJ}(-4.0 \%)$ in the OF-Bar group during the first
6 weeks and an increase in EI of 207 (SD 1490) kJ (2.1\%) in the Co-Bar group. Nevertheless, this was accompanied by a large variation between subjects and did not reach statistical significance (treatment $\times$ time: $P=0 \cdot 41$ ). Other studies found only a significant reduction of EI half-way during the studies (after 69 weeks of supplementation), but not at the end of the study (after 12-18 weeks of supplementation) for the oligofructose group compared with the control measured by a 3-d food record or an ad libitum meal ${ }^{(28,30)}$. One can speculate that the microbiota composition may be changing upon initial intervention and play a role in this reduced EI, but this effect does not persist after a longer period (7-12 weeks) of supplementation. At baseline, subjects filled out a FFQ, which was designed to quantify fibre intake ${ }^{(37)}$. The subjects reported a relatively normal fibre intake of $22 \mathrm{~g} / \mathrm{d}$, which may render them possibly less responsive to fibre supplementation. However, we did not select on fibre intake, which makes this group a normal population for this efficacy study. Their intake was marginally lower than the recommended daily intake of fibre (for adults: $25-30 \mathrm{~g} / \mathrm{d}^{(43,44)}$ ), but is somewhat higher compared with the mean intake of the average Dutch population, which is $20 \mathrm{~g} / \mathrm{d}^{(45)}$.

As expected, subjects reported significantly more flatulence in the 1 st weeks of the study, indicating colonic fermentation of oligofructose, which was also reported for other studies ${ }^{(30,46)}$. Interestingly, the level of flatulence became similar returning to baseline in both groups after about 7 weeks, suggesting an adaptation to the oligofructose ${ }^{(47)}$. However, this adaptation was not recognised in another study ${ }^{(46)}$. Despite this side effect, subjects were not aware of their treatment group; approximately $50 \%$ guessed correctly in which treatment group they were, which we assume is a matter of chance.

Major strengths of this study are the high compliance to the intervention, the realistic dosage of oligofructose and the matrix of supplementation. All participants reported to have consumed over $90 \%$ of the bars during the intervention period with an average compliance of $98 \%$. Oligofructose was added at a relatively high, but realistic, level of $16 \mathrm{~g} / \mathrm{d}$. Moreover, it was supplemented by means of a granola bar; this is in contrast to other studies in which fibres were supplemented by means of a powder in sachets dissolved in a drink ${ }^{(21-23,28-30,48)}$. In real life, it is more ecologically valid to supplement a commonly eaten food, which does not need preparation and participants can easily bring it with them, such as a granola bar.

A limitation of the present study was that subjects had only one choice of snacks for 12 weeks, and during the intervention liking of the bars decreased significantly. There is a risk that the subjects could have eaten other snacks to compensate for the boredom owing to the same flavour and texture of the bar, and consequently did not lose weight. Although the initial (non-significant) increase in flatulence and bloating, as well as effect on stool consistency, indicates that fermentation in the colon was occurring in the OF-Bar group, future studies should include serum and stool samples to measure levels of satiety hormones, SCFA or changes in microbiota composition to speculate about possible biological mechanisms.

Overall, it can be concluded that an intervention of 12-week replacement of daily snacks twice a day with an oligofructoseenriched granola bar does not have a significant effect on 
weight management outcomes when compared with replacement of daily snacks with normal granola bars. However, it seemed that satiety feelings were increased in subjects replacing their snacks for oligofructose-containing granola bars. This trial gives further insight in the use of oligofructose supplementation in weight management.

\section{Acknowledgements}

The authors would like to thank all volunteers who participated in this study. The authors further thank Henriette Fick, Desiree Lucassen, Corine Peerenboom and Nhien Ly from the division of Human Nutrition, and Melliana Jonathan from the department of Food Chemistry for their assistance with the data collection and chemical analyses.

This research was supported by Sensus BV, Roosendaal.

The authors' responsibilities were as follows: K. P. collected the data, analysed the data and wrote the manuscript; C. d. G. contributed to the design of the experiment, provided consultation and advice throughout the whole project and approved the manuscript; D. M. was involved in designing the study and writing of the protocol; M. M. designed the experiments, supervised the data collection and analyses, provided consultation and advice throughout the whole project and gave significant input to the manuscript. All authors read and approved the final version of the manuscript.

At the time of execution of the study, D. M. was an employee of Sensus BV. C. d. G. is a member of the scientific advisory board of Sensus BV. K. P. and M. M. declare that they have no conflicts of interest.

\section{Supplementary material}

For supplementary material/s referred to in this article, please visit https://doi.org/10.1017/S0007114518000211

\section{References}

1. Du H, van der A DL, Boshuizen HC, et al. (2010) Dietary fiber and subsequent changes in body weight and waist circumference in European men and women. Am J Clin Nutr 91, 329-336.

2. Liu S, Willett WC, Manson JE, et al. (2003) Relation between changes in intakes of dietary fiber and grain products and changes in weight and development of obesity among middle-aged women. Am J Clin Nutr 78, 920-927.

3. Viskaal-van Dongen M, Kok FJ \& de Graaf C (2011) Eating rate of commonly consumed foods promotes food and energy intake. Appetite 56, 25-31.

4. Holt SHA, Miller JCB, Petocz P, et al. (1995) A satiety index of common foods. Eur J Clin Nutr 49, 675-690.

5. Clark MJ \& Slavin JL (2013) The effect of fiber on satiety and food intake: a systematic review. J Am Coll Nutr 32, 200-211.

6. Wanders AJ, van den Borne JJGC, de Graaf C, et al. (2011) Effects of dietary fibre on subjective appetite, energy intake and body weight: a systematic review of randomized controlled trials. Obes Rev 12, 724-739.

7. Poutanen KS, Dussort P, Erkner A, et al. (2017) A review of the characteristics of dietary fibers relevant to appetite and energy intake outcomes in human intervention trials. Am J Clin Nutr 106, $747-754$.
8. Coussement PAA (1999) Inulin and oligofructose: safe intakes and legal status. J Nutr 129, 1412S-1417S.

9. Mensink MA, Frijlink HW, van der Voort Maarschalk K, et al. (2015) Inulin, a flexible oligosaccharide I: Review of its physicochemical characteristics. Carbohydr Polym 130, 405-419.

10. Roberfroid MB (1999) Caloric value of inulin and oligofructose. J Nutr 129, 1436S-1437S.

11. Roberfroid MB (2005) Introducing inulin-type fructans. $\mathrm{Br} J$ Nutr 93, S13-S25.

12. Slavin J (2013) Fiber and prebiotics: mechanisms and health benefits. Nutrients 5, 1417-1435.

13. Roberfroid M, Gibson GR, Hoyles L, et al. (2010) Prebiotic effects: metabolic and health benefits. Br J Nutr 104, S1-S63.

14. Cani PD, Dewever C \& Delzenne NM (2004) Inulin-type fructans modulate gastrointestinal peptides involved in appetite regulation (glucagon-like peptide-1 and ghrelin) in rats. Br J Nutr $\mathbf{9 2}, 521-526$.

15. Cani PD, Neyrinck AM, Maton N, et al. (2005) Oligofructose promotes satiety in rats fed a high-fat diet: involvement of glucagon-like peptide-1. Obes Res 13, 1000-1007.

16. Cani PD, Knauf C, Iglesias MA, et al. (2006) Improvement of glucose tolerance and hepatic insulin sensitivity by oligofructose requires a functional glucagon-like peptide 1 receptor. Diabetes 55, 1484-1490.

17. Cani PD, Hoste S, Guiot Y, et al. (2007) Dietary non-digestible carbohydrates promote L-cell differentiation in the proximal colon of rats. Br J Nutr 98, 32-37.

18. Dewulf EM, Cani PD, Neyrinck AM, et al. (2011) Inulin-type fructans with prebiotic properties counteract GPR43 overexpression and PPAR $\gamma$-related adipogenesis in the white adipose tissue of high-fat diet-fed mice. J Nutr Biochem 22, 712-722.

19. Arora T, Loo RL, Anastasovska J, et al. (2012) Differential effects of two fermentable carbohydrates on central appetite regulation and body composition. PLOS ONE 7, e43263.

20. Hadri Z, Chaumontet C, Fromentin G, et al. (2015) Long term ingestion of a preload containing fructo-oligosaccharide or guar gum decreases fat mass but not food intake in mice. Physiol Behav 147, 198-204.

21. Cani PD, Joly E, Horsmans Y, et al. (2006) Oligofructose promotes satiety in healthy human: a pilot study. Eur J Clin Nutr 60, 567-572.

22. Cani PD, Lecourt E, Dewulf EM, et al. (2009) Gut microbiota fermentation of prebiotics increases satietogenic and incretin gut peptide production with consequences for appetite sensation and glucose response after a meal. Am J Clin Nutr 90, 1236-1243.

23. Pedersen C, Lefevre S, Peters V, et al. (2013) Gut hormone release and appetite regulation in healthy non-obese participants following oligofructose intake. A dose-escalation study. Appetite 66, 44-53.

24. Verhoef SPM, Meyer D \& Westerterp KR (2011) Effects of oligofructose on appetite profile, glucagon-like peptide 1 and peptide YY3-36 concentrations and energy intake. Br J Nutr 106, 1757-1762.

25. Hess JR, Birkett AM, Thomas W, et al. (2011) Effects of shortchain fructooligosaccharides on satiety responses in healthy men and women. Appetite 56, 128-134.

26. Peters HP, Boers HM, Haddeman E, et al. (2009) No effect of added $\beta$-glucan or of fructooligosaccharide on appetite or energy intake. Am J Clin Nutr 89, 58-63.

27. Karalus M, Clark M, Greaves KA, et al. (2012) Fermentable fibers do not affect satiety or food intake by women who do not practice restrained eating. J Acad Nutr Diet 112, 1356-1362.

28. Parnell JA \& Reimer RA (2009) Weight loss during oligofructose supplementation is associated with decreased ghrelin and increased peptide YY in overweight and obese adults. $\mathrm{Am}$ J Clin Nutr 89, 1751-1759. 
29. Daud NM, Ismail NA, Thomas EL, et al. (2014) The impact of oligofructose on stimulation of gut hormones, appetite regulation, and adiposity. Obesity 22, 1430-1438.

30. Guess ND, Dornhorst A, Oliver N, et al. (2015) A randomized controlled trial: the effect of inulin on weight management and ectopic fat in subjects with prediabetes. Nutr Metab (Lond) 12, 36.

31. van Strien T, Frijters JER, Bergers GPA, et al. (1986) The Dutch eating behavior questionnaire (DEBQ) for assessment of restrained, emotional and external eating behavior. Int J Eat Disord 5, 295-315.

32. World Health Organization (1985) Principles for the Estima tion of Energy Requirements. Energy and Protein Require ments. Report of a Joint FAO/WHO/UNU Expert Consultation. Report of a Joint FAO/WHO/UNU Expert Consultation, 2nd ed. Geneva: WHO.

33. Ainsworth BE, Haskell WL, Herrmann SD, et al. (2011) 2011 Compendium of Physical Activities: a second update of codes and MET values. Med Sci Sports Exerc 43, 1575-1581.

34. Brožek J, Grande F, Anderson JT, et al. (2006) Densitometric analysis of body composition: revision of some quantitative assumptions*. Ann N Y Acad Sci 110, 113-140.

35. Fields DA, Goran MI \& McCrory MA (2002) Body-composition assessment via air-displacement plethysmography in adults and children: a review. Am J Clin Nutr 75, 453-467.

36. Bosy-Westphal A, Danielzik S, Becker C, et al. (2005) Need for optimal body composition data analysis using air-displacement plethysmography in children and adolescents. J Nutr 135, 2257-2262.

37. Wanders AJ, Mars M, Borgonjen-van den Berg KJ, et al. (2014) Satiety and energy intake after single and repeated exposure to gel-forming dietary fiber: post-ingestive effects. Int J Obes (Lond) 38, 794-800.

38. Tucker P \& Gilliland J (2007) The effect of season and weather on physical activity: a systematic review. Public Health 121, 909-922.

39. Bel-Serrat S, Julián-Almárcegui $\mathrm{C}$, González-Gross $\mathrm{M}$, et al. (2016) Correlates of dietary energy misreporting among
European adolescents: the Healthy Lifestyle in Europe by Nutrition in Adolescence (HELENA) study. Br J Nutr 115, 1439-1452.

40. Winkels RM, Jolink-Stoppelenburg A, de Graaf K, et al. (2011) Energy intake compensation after 3 weeks of restricted energy intake in young and elderly men. J Am Med Dir Assoc 12, 277-286.

41. Goris AH, Westerterp-Plantenga MS \& Westerterp KR (2000) Undereating and underrecording of habitual food intake in obese men: selective underreporting of fat intake. Am J Clin Nutr 71, 130-134.

42. Poslusna K, Ruprich J, de Vries JH, et al. (2009) Misreporting of energy and micronutrient intake estimated by food records and 24 hour recalls, control and adjustment methods in practice. Br J Nutr 101, Suppl. 2, S73-S85.

43. European Food Safety Authority, Panel on Dietetic Products, Nutrition and Allergies (NDA) (2010) Scientific opinion on dietary reference values for carbohydrates and dietary fibre. EFSA J 8, 1462-1539.

44. Scientific Advisory Committee on Nutrition (2015) Carbohydrates and Health. London: TSO.

45. National Institute for Public Health and the Environment (RIVM) (2013) Dutch National Food Consumption Survey 2007-2010. Diet of Children and Adults Aged 7 to 69 Years Version 2, Based on Dataset DFC_2010_core_20120822. Bilthoven: RIVM.

46. Dahl WJ, Wright AR, Specht GJ, et al. (2014) Consuming foods with added oligofructose improves stool frequency: a randomised trial in healthy young adults. J Nutr Sci $\mathbf{3}$, e7.

47. Kolida S, Meyer D \& Gibson GR (2007) A double-blind placebo-controlled study to establish the bifidogenic dose of inulin in healthy humans. Eur J Clin Nutr 61, 1189-1195.

48. Liber A \& Szajewska H (2014) Effect of oligofructose supplementation on body weight in overweight and obese children: a randomised, double-blind, placebo-controlled trial. BrJ Nutr 112, 2068-2074. 